\title{
Full-length transcriptome analysis of shade- induced promotion of tuber production in Pinellia ternata
}

Tao Xue ${ }^{\dagger}$, Han Zhang ${ }^{\dagger}$, Yuanyuan Zhang, Shuqin Wei, Qiujie Chao, Yanfang Zhu, Jingtong Teng, Aimin Zhang, Wei Sheng, Yongbo Duan * (D) and Jianping Xue*

\begin{abstract}
Background: Pinellia ternata is native to China and has been used as a traditional herb due to its antiemetic, antitussive, analgesic, and anxiolytic effects. When exposed to strong light intensity and high temperature during the reproductive growth process, $P$. ternata withers in a phenomenon known as "sprout tumble", which largely limits tuber production. Shade was previously found to delay sprout tumble formation (STF); however, no information exists regarding this process at the molecular level. Hence, we determined the genes involved in tuber development and STF in P. ternata.

Results: Compared to that with natural sun-light (control), shade significantly induced chlorophyll accumulation, increased chlorophyll fluorescence parameters including initial fluorescence, maximal fluorescence, and $q P$, and dramatically repressed chlorophyll a:b and NPQ. Catalase (CAT) activity was largely induced by shade, and tuber products were largely increased in this environment. Transcriptome profiles of $P$. ternata grown in natural sun-light and shaded environments were analyzed by a combination of next generation sequencing (NGS) and third generation single-molecule real-time (SMRT) sequencing. Corrections of SMRT long reads based on NGS short reads yielded 136,163 non-redundant transcripts, with an average N50 length of 2578 bp. In total, 6738 deferentiallyexpressed genes (DEGs) were obtained from the comparisons, specifically D5S vs D5CK, D20S vs D20CK, D20S vs D5S, and D20CK vs D5CK, of which, 6384 DEGs (94.8\%) were generated from the D20S vs D20CK comparison. Gene annotation and functional analyses revealed that these genes were related to auxin signal transduction, polysaccharide and sugar metabolism, phenylpropanoid biosynthesis, and photosynthesis. Moreover, the expression of genes enriched in photosynthesis appeared to be significantly altered by shade. The expression patterns of 16 candidate genes were consistent with changes in their transcript abundance as identified by RNA-Seq, and these might contribute to STF and tuber production.
\end{abstract}

Conclusion: The full-length transcripts identified in this study have provided a more accurate depiction of $P$. ternata gene transcription. Further, we identified potential genes involved in STF and tuber growth. Such data could serve as a genetic resource and a foundation for further research on this important traditional herb.

Keywords: Pinellia ternata, Shade, Transcriptome, Single-molecule real-time sequencing, Sprout tumble, Tuber production

\footnotetext{
* Correspondence: yboduan@163.com; xuejp@163.com

${ }^{\dagger}$ Tao Xue and Han Zhang contributed equally to this work.

Key Laboratory of Resource Plant Biology of Anhui Province, College of Life

Sciences, Huaibei Normal University, Huaibei 235000, China
}

(c) The Author(s). 2019 Open Access This article is distributed under the terms of the Creative Commons Attribution 4.0 International License (http://creativecommons.org/licenses/by/4.0/), which permits unrestricted use, distribution, and reproduction in any medium, provided you give appropriate credit to the original author(s) and the source, provide a link to the Creative Commons license, and indicate if changes were made. The Creative Commons Public Domain Dedication waiver (http://creativecommons.org/publicdomain/zero/1.0/) applies to the data made available in this article, unless otherwise stated. 


\section{Background}

Pinellia ternata belongs to the Araceae family and is a perennial herb that is widely distributed in the eastern part of Asia. Further, both its wild and cultivated varieties are mainly found in China [1,2]. Its tuber is the main medicinal part, and this has been frequently used in traditional Chinese medicine for thousands of years [3-5]. Many studies have revealed the complex components of $P$. ternata, such as alkaloids, organic acid, polysaccharose, proteins, and nucleosides [6-11]. Alkaloid has however been recognized as its main active ingredient and is believed to exert anticancer effects $[12,13]$. Besides this property, the antiemetic, antitussive, analgesic, and anxiolytic effects of $P$. ternata are valuable for its use as a traditional Chinese medicine $[9,10,14,15]$. As the lectin protein in the tuber of $P$. ternata displays toxic effects [16], the development of an eco-friendly biopesticide using the extracts of this plant and the breeding of diseaseresistant varieties using agglutinin gene engineering are warranted [17-19]. Although the current demand for P. ternata is growing, sources of the plant are becoming increasingly scarce due to over-exploitation and the lack of large-scale cultivation.

P. ternata is sensitive to light intensity and temperature during growth. When exposed to strong light and high temperature, it rapidly withers, forming a "sprout tumble" [20]. The "sprout tumble" often appears in the summer and directly limits tuber production, which exacerbates the disparities between the supply and demand of P. ternata [21]. Hence, reducing sprout tumble formation (STF) is becoming a key procedure to increase tuber production. The rate and date of STF in P. ternata can be dramatically remitted under shading conditions during its reproductive growth period and this has been revealed at the physiological level [22-24]. To date, however, a report revealing this mechanism at the molecular level has not been presented, thereby warranting the need for a complete understanding of the effect of shade on STF.

With the rapid development of molecular biology and bioinformatics, gene discovery can be performed in non-model species, especially for non-sequenced medicinal plants [25-27]. For P. ternata, candidate genes might be involved in tuber development, and its high temperature-response has been verified via EST library sequencing [20]. Genes related to the ephedrine biosynthetic pathway were previously revealed by next-generation sequencing (NGS) [28]. Although NGS is currently used to generate large amounts of omics data [29, 30], identifying gene isoforms based on the short reads generated from NGS could be hindered by a high incidence of false positives [31]. Single-molecule real-time (SMRT) sequencing was recently demonstrated to offer a great advantage over NGS technologies in terms of read length [32] and has since been used to characterize the complexity of transcriptomes in Arabidopsis pumila, Hordeum vulgare, and Camellia sinensis [3335]. As mentioned, the genes that regulate STF respond to shade, whereas those that regulate tuber production are largely unknown. In this study, we combined NGS and SMRT sequencing technologies to identify the full-length transcriptome response of $P$. ternata to shade, as well as candidate genes involved in STF and tuber development. This work serves as a foundation to functionally elucidate the genes involved in tuber development and STF in $P$. ternata.

\section{Results}

Shade inhibits the sprout tumble rate and promotes tuber production

To investigate the effect of shade on $P$. ternata, we evaluated the rate of STF in $P$. ternata grown under control and shade conditions. Almost all P. ternata plants grown in control conditions showed STF, but this rate was sharply decreased in the shade (Fig. 1a). Meanwhile, the tubers generated from $P$. ternata grown in the shade weighed $\sim 14 \mathrm{~g}$ per seedling, 2-fold greater than the control weight (Fig. $1 \mathrm{~b}$ and d). We next sought to detect the physiological traits of P. ternata, and as shown in Table 1, the contents of chlorophyll a and chlorophyll b increased while the chlorophyll a:b ratio decreased in the shade compared to those in the control. Values of initial fluorescence $\left(F_{0}\right)$, maximal fluorescence $\left(F_{m}\right)$, and $q P$ were elevated in the shade environment, whereas $N P Q$ was reduced (Table 1). We also evaluated the enzyme activity of superoxide dismutase (SOD), catalase (CAT), and polyamine oxidase (POD) under control and shade conditions. CAT activity was largely increased in $P$. ternata plants grown in the shade. However, statistical significance was not found for SOD and POD activities between control and shade conditions (Fig. 2).

\section{Transcriptome sequencing}

To identify and characterize the transcriptomes of $P$. ternata, between control and shade environments, NGS and SMRT sequencing technologies were employed for whole-transcriptome profiling. More than 617 million clean reads were generated by Illumina sequencing (Additional file 1: Table S1). In addition, SMRT generated 498,369 insert reads, of which 297,364 were fulllength non-chimeric (flnc) reads and 127,681 were nonfull-length reads (Table 2). The average flnc read length was 2306 bp (Additional file 2: Table S2). 

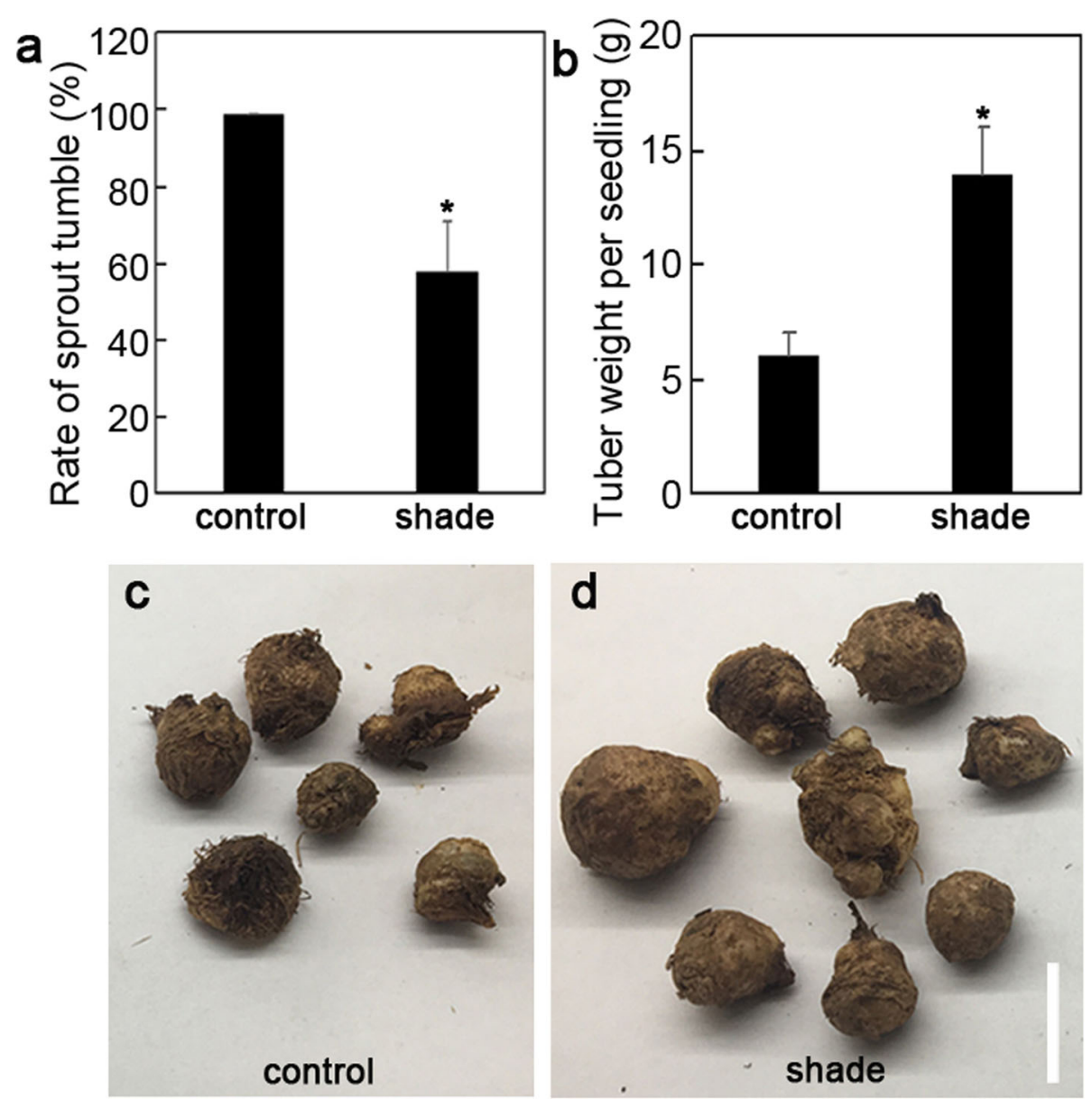

Fig. 1 Effect of shade on the growth of $P$. ternata. Effect of shade on the rate of STF (a) and tuber weight (b) of $P$. ternata grown in a control and a shaded environment. Tuber weight of $P$. ternata grown in control and shade environment. Tubers harvested in control (c) and shaded environment (d) after all $P$. ternata had STF. Data are presented as mean \pm SD $(n>30)$. * indicates significant difference $(P<0.05)$

To reduce the high error rates of the subreads, all SMRT reads were corrected using the $\sim 617$ million Illumina clean reads as input data (Additional file 1: Table S1). After error correction and the elimination of redundant transcripts using the CD-HIT program, 136,163 non-redundant transcripts were generated, each of which represented a unique full-length transcript with an average length of 2348 bp and N50 of 2578 bp (Additional file 2: Table S2). Approximately 83,075 unigenes (61\%) were longer than 2 $\mathrm{kb}$ and only 13,074 unigenes (9.6\%) were shorter than 500

Table 1 Photosynthetic parameter of $P$. ternata under control and shade environments

\begin{tabular}{|c|c|c|}
\hline \multirow[t]{2}{*}{ Photosynthetic parameter } & \multicolumn{2}{|l|}{ Light condition } \\
\hline & Control & Shade \\
\hline Chlorophyll a content (mg. $\left.\mathrm{g}^{-1} \mathrm{DM}\right)$ & $3.07 \pm 0.17$ & $4.88 \pm 0.32^{*}$ \\
\hline Chlorophyll b content (mg. g ${ }^{-1}$ DM) & $1.05 \pm 0.11$ & $2.11 \pm 0.18^{*}$ \\
\hline Chlorophyll $(a+b)$ content $\left(\mathrm{mg} \cdot \mathrm{g}^{-1} \mathrm{DM}\right)$ & $4.12 \pm 0.28$ & $6.99 \pm 0.49^{*}$ \\
\hline Chlorophylla:b & $2.93 \pm 0.15$ & $2.32 \pm 0.08^{*}$ \\
\hline Initial fluorescence $\left(F_{0}\right)$ & $219.54 \pm 13.61$ & $247.33 \pm 11.90^{*}$ \\
\hline Maximal fluorescence $\left(F_{m}\right)$ & $1153.46 \pm 162.29$ & $1240.17 \pm 6.39^{*}$ \\
\hline$q P$ & $0.91 \pm 0.039$ & $0.97 \pm 0.012^{*}$ \\
\hline$N P Q$ & $0.027 \pm 0.005$ & $0.016 \pm 0.008^{*}$ \\
\hline
\end{tabular}

Data are presented as means $\pm \mathrm{sd}$ from three replicates with 30 explants in each replicate. ${ }^{*}$ indicates significant difference between control and shade $(P<0.05)$ 

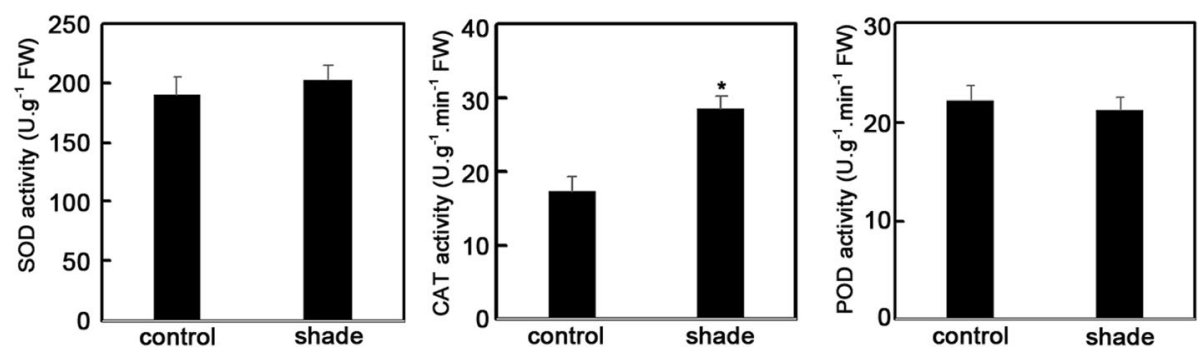

Fig. 2 Effect of the 20-d-shade treatment on SOD, CAT, and POD enzymatic activity in $P$. ternata. Data are presented as mean $\pm S D(n>30)$. * indicates significant difference $(P<0.05)$

bp (Fig. 3). Altogether, the SMRT sequencing technology provided a high-quality transcript profile with many fulllength genes in P. ternata.

\section{Functional annotation and categorization of genes}

To acquire a comprehensive annotation profile, NCBI non-redundant protein (NR), Kyoto Encyclopedia of Genes and Genomes (KEGG), Protein family (Pfam), Swiss-Prot, EuKaryotic Ortholog Groups (KOG), Gene Ontology (GO) by BLASTX, and NCBI nucleotide sequences (NT) by BLASTN were used to align the 13,613 transcripts. A total of 115,708 (84.98\%) genes were successfully annotated using these databases. A Venn diagram showed that 43,106 genes were simultaneously annotated by NR, GO, Pfam, KOG, and NT (Additional file 3: Fig. S1A). Based on the alignment of sequences from different species in the NR database, 79,607 (68.8\%) sequences had significant hits for Anthurium amnicola, followed by Elaeis guineensis (5207, 4.5\%), Phoenix dactylifera (3703, 3.2\%), Nelumbo nucifera (2314, 2.0\%), and Ananas comosus (2083, 1.8\%). Only $19.7 \%$ of the annotated sequences were identified based on sequences in other plant species (Additional file 3: Fig. S1B).

Various TF families including the WRKY, NAC, and MYB families were confirmed to be involved in the

Table 2 Statistics of SMRT sequencing data

\begin{tabular}{ll}
\hline Library & Number \\
\hline Number of SMRT cells & 2 \\
Number of reads of insert & 498,369 \\
Number of 5' reads & 418,110 \\
Number of 3' reads & 444,553 \\
Number of ploy-A reads & 430,421 \\
Number of filtered short reads & 21,211 \\
Number of non-full-length reads & 127,681 \\
Number of full-length reads & 349,477 \\
Number of full-length non-chimeric reads & 297,364 \\
Average full-length non-chimeric read length & 2306 \\
\hline
\end{tabular}

regulation of plant growth and their response to the environment. Transcriptome analysis of $P$. ternata revealed that 7783 genes $(5.7 \%)$ encode putative TFs that can be classified into the 28 major TF families, as well as some other families. Members of the C3H, WRKY, and bHLH TF families occupied the top three slots, and all exceeded 400. The number of GRAS, AP2/ERF, bZIP, $\mathrm{C} 2 \mathrm{H} 2$, NAC, MYB-related, HSF, and B3 families exceeded 200 (Additional file 4: Fig. S2). The identification of this large set of TFs provides a rich resource for the further analysis of specific TFs in various life processes of $P$. ternata.

To functionally classify $P$. ternata genes, they were mapped to various terms of the GO database (http:// www.geneontology.org/). A total of 73,250 genes were mainly classified into three major categories (biological process, cellular component, and molecular function). Based on molecular function classification, major categories were "binding" and "catalytic activity". The major subgroups for cellular components were "cell", "cell part", "organelle", "membrane", and "membrane part". For the biological process category, genes involved in the "metabolic process", "cellular process", and "single-organism process" were highly represented (Additional file 5: Fig. S3).

To understand the functions in a specific metabolic pathway of $P$. ternata, we assigned the assembled genes to KEGG biological pathways. Consequently, functions could be assigned to five main categories as follows: "Cellular Processes", "Environmental Information Processing", "Genetic Information Processing", "Metabolism", and "Organismal Systems", with 33 sub-categories. Among these categories, "signal transduction" had 6757 genes, "carbohydrate metabolism" had 5925 genes, and "endocrine system" had 3462 genes, which provides valuable information for further gene function analysis (Additional file 6: Fig. S4).

\section{Analysis of differentially-expressed genes (DEGs)}

To evaluate gene expression levels in response to shade, Illumina clean reads of control and shade samples assembled with the SMRT full-length transcriptome were 


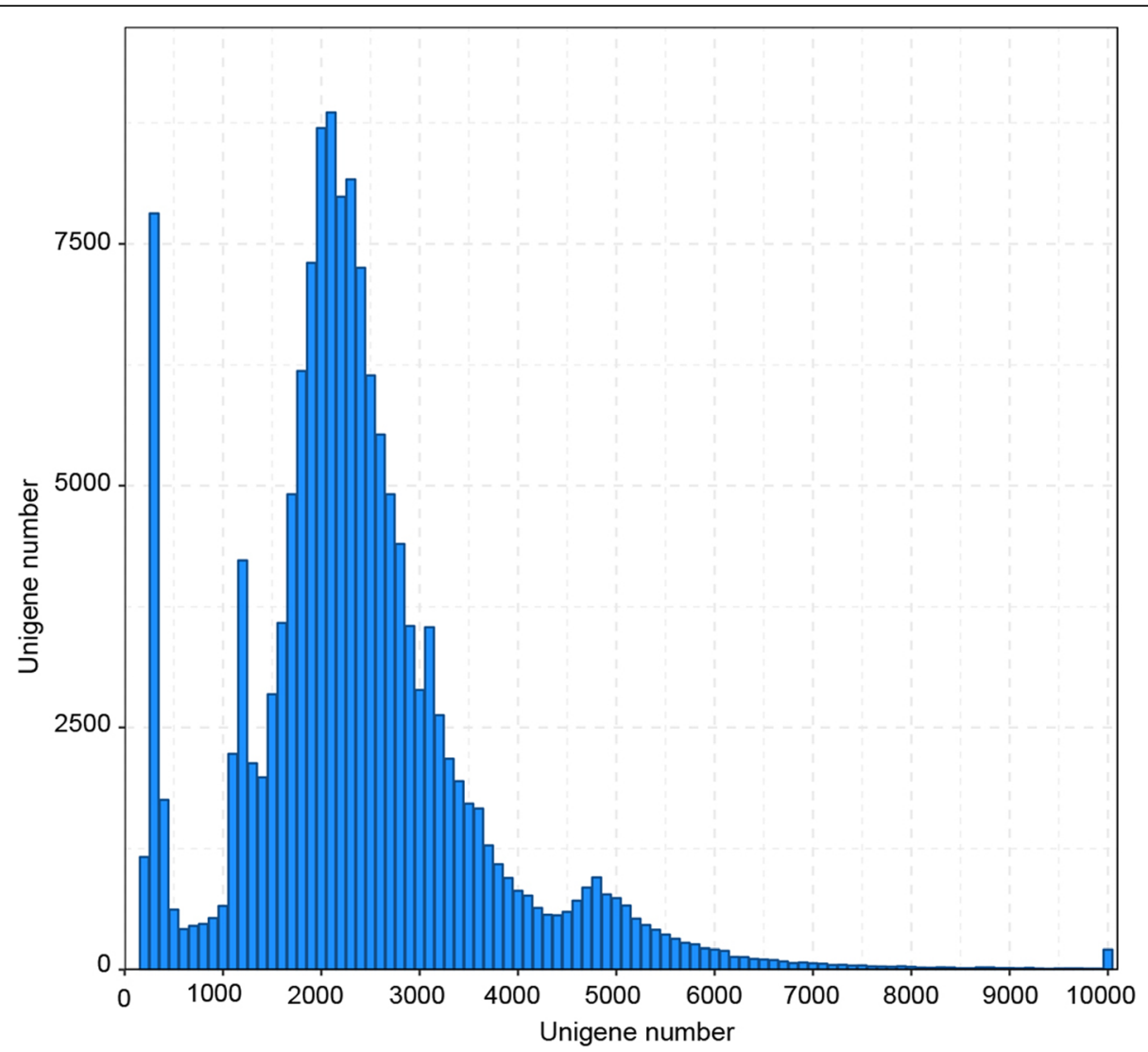

Fig. 3 Length distribution of the $P$. ternata transcripts

mapped. Thereafter, read-counts for each gene were obtained from the mapping results, and then converted into an expected number of fragments per kilobase of transcript sequence per million base pairs (FPKM).

In total, 6738 DEGs that were up or downregulated between samples $(P<0.05)$ were collected. Clustering patterns of DEGs under different experimental treatments were determined by the cluster analysis of all DEGs with the Euclidean distance method and complete linkage (Fig. 4). This clustering pattern suggested that many genes were activated or inhibited in the D20S vs D20CK comparison, and the number of DEGs were much greater than those with the D5S vs D5CK, D20S vs D5S, and D20CK vs D5CK comparisons (Additional file 7: Fig. S5).

To gain a better understanding of DEG functions, we selectively analyzed the 6384 DEGs from the D20S vs D20CK comparison. Based on GO functional enrichment analysis, these DEGs could be classified into two categories, namely biological processes and molecular functions, with 30 functional groups. For the molecular function ontology, the dominant terms were "heterocyclic compound binding", "organic cyclic compound binding", and "ion binding". For biological processes, 15 functional groups had almost equivalent gene numbers without dominant terms (Fig. 5). KEGG pathway enrichment analysis was also carried out and DEGs could be assigned to 112 KEGG pathways. Here, we focused on the top 30 pathways and screened four that are closely related to STF and tuber growth, namely "photosynthesis-antenna proteins", "phenylpropanoid biosynthesis", "plant hormone signal transduction", and "starch and sucrose metabolism" (Fig. 6).

\section{qRT-PCR validation of candidate genes involved in STF and tuber growth}

To validate the transcriptome data, 16 candidate DEGs that could be associated with STF and tuber growth were selected for qRT-PCR analysis. As shown in Fig. 7, DEGs involved in antioxidant protection $(S O D, C A T$, $P O D$, and GR), auxin signal transduction (ARF1, ARF19, PIN1, and PIN6), polysaccharide and sugar metabolism (XET, RGP, and SUS), and phenylpropanoid biosynthesis $(C H S)$ were upregulated. DEGs related to photosynthesis (PIF3, CAB151, and RUB) were also upregulated but HY5 was downregulated in the 20-d-shade environment. Moreover, fold-changes in their expression, which were calculated by sequencing did not match the expression 


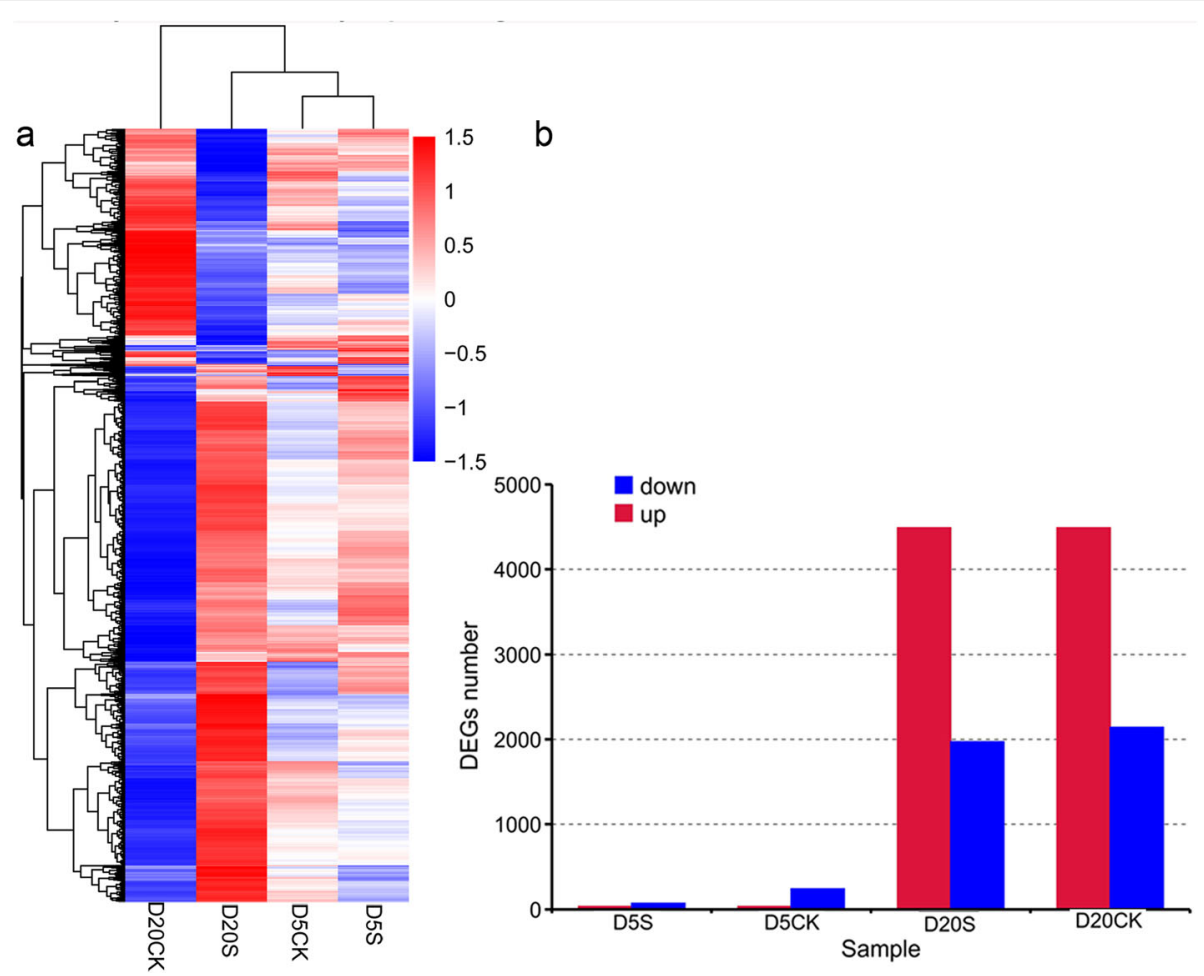

Fig. 4 Clustering analysis of the DEGs. a Hierarchical clustering graph of the 6738 DEGs based on average $\log _{10}($ FPKM+1) values of all genes in each cluster. $\mathbf{b}$ statistics of upregulated- and downregulated-expression of DEGs in each cluster

data detected by qRT-PCR. However, the expression trends were in agreement for these 16 genes. We also analyzed expression of these genes after growing $P$. ternata in the shade environment for $5 \mathrm{~d}$. Compared to control levels, the expression of most genes showed no significant changes, although several genes (PtPIF3, PtSOD, PtCAT, PtPOD, PtARF1, PtXET, and PtRGP) were upregulated (Fig. 7). The expression profiles thus

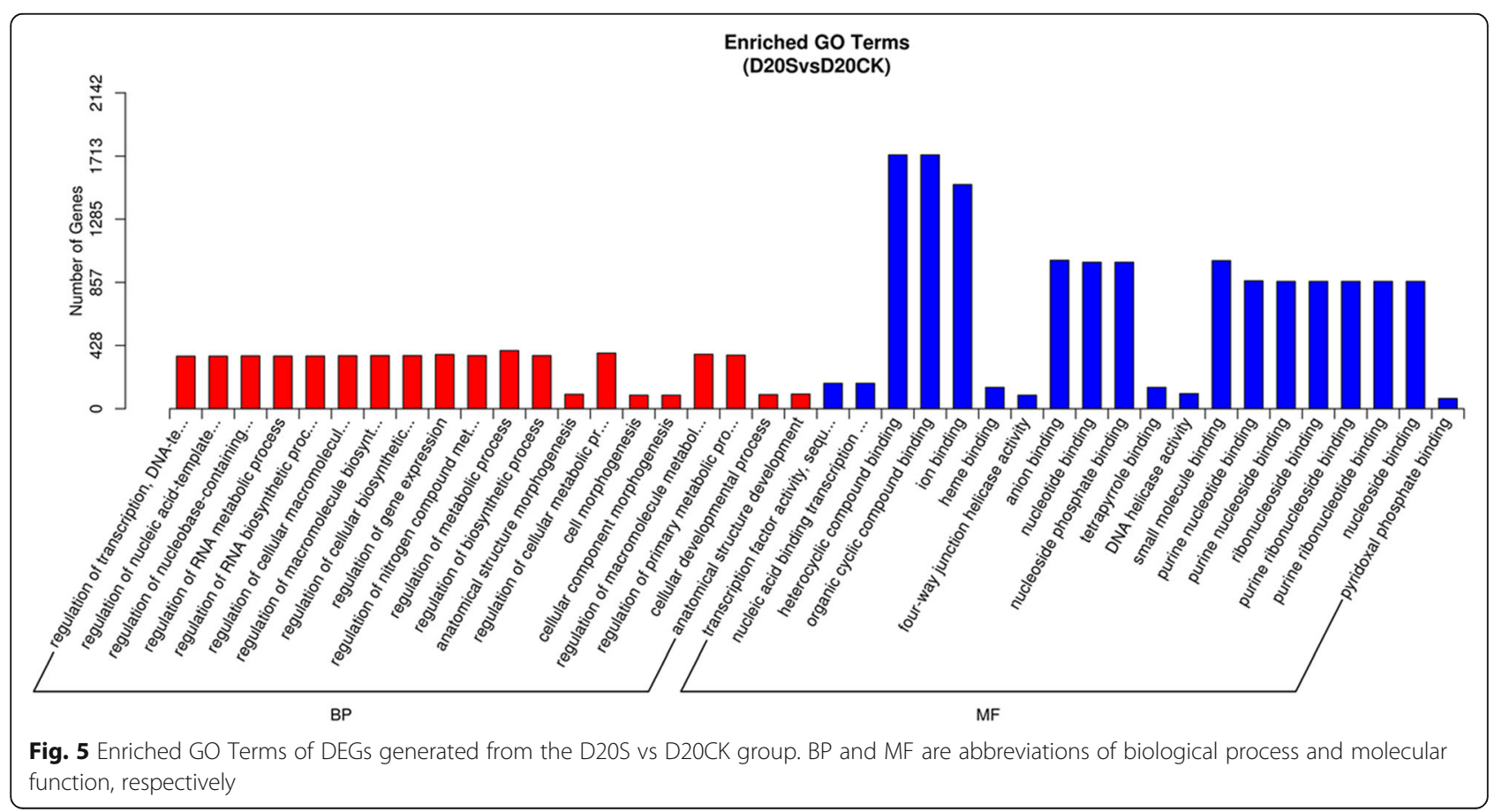




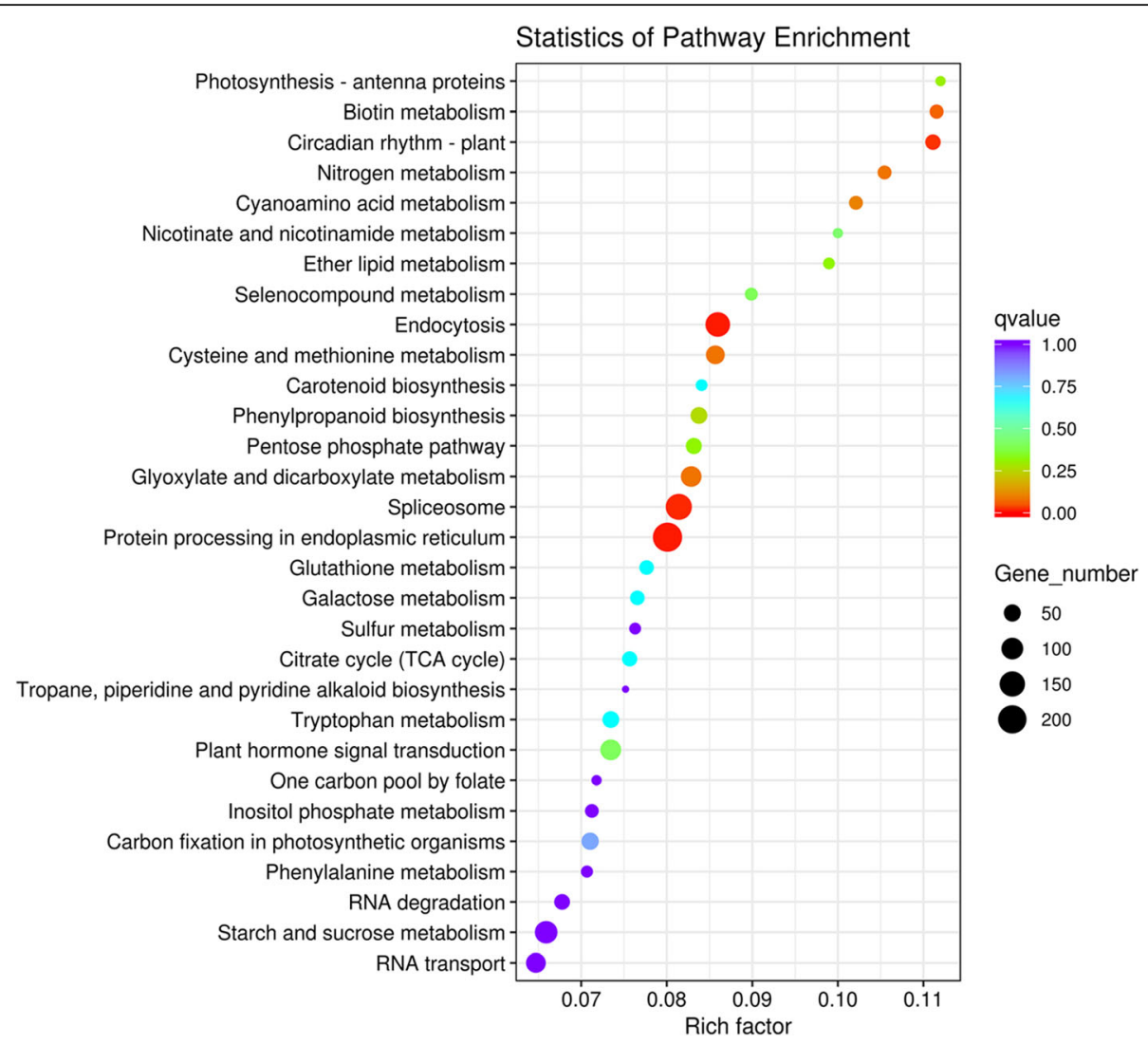

Fig. 6 KEGG enrichment analyses with the DEGs generated from the D20S vs D20CK group. The rich factor is the ratio of differentially expressed genes versus all annotated genes in corresponding pathways

confirmed the reliability of the transcriptomic data and provided useful information to understand STF and tuber growth in P. ternata.

\section{Discussion}

Plant growth and development often correlate with environmental factors. Two important factors, light and temperature, always regulate similar developmental processes in the lifecycle of plants [36, 37]. High temperature along with strong light often causes STF in P. ternata [20]. Here, we revealed that growth in a shaded environment promotes tuber production by inhibiting the rate of STF, which aligns with the findings of a previous report [22].

Recently, some researchers assessed P. ternata and explored some of its transcript profiles for hightemperature stress [20], in vitro tuber induction [37], and the ephedrine biosynthetic pathway [28]. However, the genetic background remains largely unknown, thereby prohibiting further research and improvements in P. ternata cultivation. Currently, the use of a hybrid sequencing approach where NGS and SMRT are combined is gradually increasing, which could provide highquality and complete assemblies, especially with nonsequenced species [38-40]. In this study, a more complete transcriptome of $P$. ternata was generated by using NGS and SMRT technologies. Corrections of the SMRT reads using the NGS reads generated highquality, full-length reads, largely reducing the misassemblies of genes with high sequence identity. Such high-quality data have aided in the understanding of STF and tuber growth and have allowed for future studies on P. ternata.

By comparing DEGs generated in different groups, we found that DEGs in the D20S vs D20CK comparison accounted for 94.12\% (Additional file 7: Fig. S5). This result suggests that a long duration of shade treatment might be the key factor promoting tuber development through the altered expression of many genes. The 112 KEGG pathways involved in DEGs revealed that STF and tuber growth in $P$. ternata are intricate life phenomena (Fig. 6). In the shaded condition, photosynthetic traits of $P$. ternata including chlorophyll content and chlorophyll fluorescence parameters were significantly increased (Table 1), demonstrating that this condition improves photosynthesis, which aligns with previous data obtained in our lab [41]. Similarly, the transcript of PtPIF3, which encodes a suppressor of photosynthesis, was found to increase while that of the positive regulator 

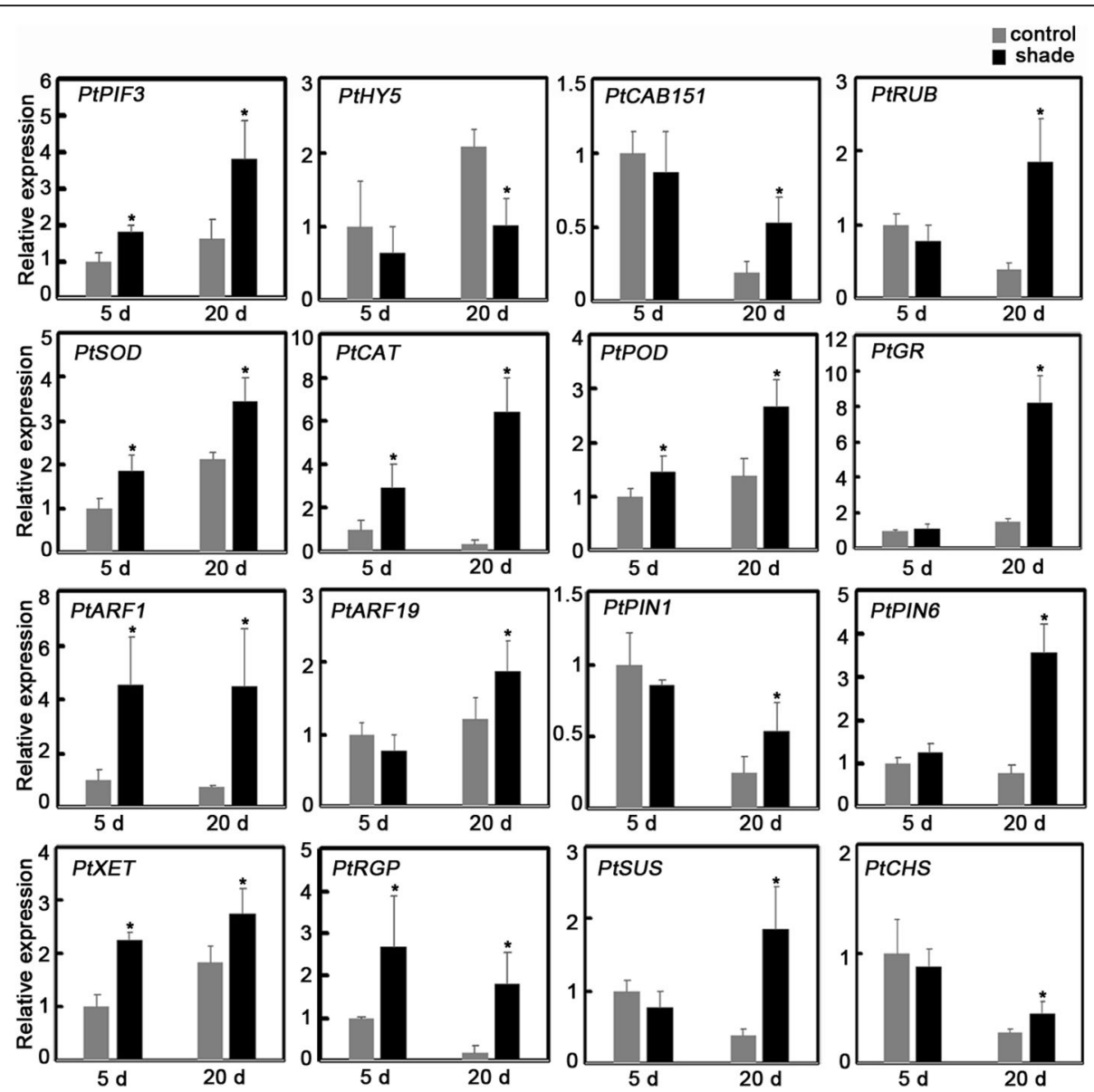

Fig. 7 The 16 genes with differential expression when $P$. ternata is grown for 5 and $20 \mathrm{~d}$ in control and shaded environment, respectively. All the data represent the values relative to those of control at $5 \mathrm{~d}$. Data are presented as mean $\pm \mathrm{SD} .{ }^{*}$ means differed significantly $(P<0.05)$. PIF3: phytochrome-interacting factor 3; HY5: elongated hypocotyls 5; CAB151: chlorophyll a/b-binding proteins 151; RUB: rubisco; SOD: superoxide dismutase; CAT: catalase; POD: polyamine oxidase; GR: glutathione reductase; ARF1/19: auxin response factor 1/19; PIN1/6: PIN FORMED1/6; XET: $\beta$-xyloglucan endotransglycosylase; RGP: reversible glycosylated polypeptides; SUS: sucrose synthase; and CHS: chalcone synthase

PtHY5 decreased after $20 \mathrm{~d}$ of shade treatment (Fig. 7) [42]. This indicates that the light signaling pathway is greatly altered in the shade environment. Furthermore, the transcript abundances of PtCAB151 and PtRUB markedly increased in the 20-d shade environment (Fig. 7) $[43,44]$. Therefore, by combining physiological and genetic data, photosynthetic efficiency was found to increase to capture the limited light resource $[45,46]$; this result aligns with that of a previous study where soybean photosynthesis was found to be enhanced in a shaded environment [47]. Only PtPIF3 was upregulated in $P$. ternata after $5 \mathrm{~d}$ of shade treatment. This demonstrated that the photosystem of $P$. ternata was affected at day 5 and was amplified after a $20-\mathrm{d}$ shade treatment.

Higher plants use light energy for photosynthesis. However, this always leads to photoinhibition when the light energy absorbed by pigments exceeds the capacity of the photosynthetic apparatus [48]. This can then lead to ROS production, which damages the photosynthetic system $[49,50]$. Based on oxidative stress, relatedenzymes, mainly SOD, CAT, POD, and glutathione reductase (GR), can be induced to eliminate ROS [50-54]. Previous data demonstrated that $P$. ternata easily underwent photoinhibition when it was exposed to strong light in the summer [55]. Here, transcripts of PtSOD, PtCAT, and PtPOD were increased after a 5 -d shade treatment, and the expression of PtSOD, PtCAT, PtPOD, and $P t G R$ were upregulated after a $20-\mathrm{d}$ shade treatment (Fig. 7). The activity of PtCAT dramatically increased in the shaded environment, whereas the activities of PtSOD and PtPOD showed no significant changes (Fig. 2). These findings imply that shading treatment protects $P$. ternata from ROS damage by regulating CAT activity, which delays STF. It was speculated that the activities of PtSOD and PtPOD were further regulated at the translation level, thereby reducing the differences in genes expression, as required for precise regulation. Notably, $\mathrm{CHS}$ is a key enzyme in flavonoid synthesis and it is 
involved in the phenylalanine synthesis pathway and the regulation of plant resistance to adversity [56]. Our study showed that the expression level of PtCHS was strongly induced in the 20-d shade environment (Fig. 7), aligning with a previous report showing that light suppresses $\mathrm{PtCHS}$ accumulation, thereby inhibiting sweet potato growth [57]. The implication is that high PtCHS expression might promote tuber growth in P. ternata; however, whether it participates in the regulation of STF requires further verification.

Through exposure to a shaded environment, $P$. ternata plants grow faster, with high seedling heights and enlarged leaf and tuber size [22]. Plant growth requires cell proliferation and enlargement, which are closely-related to cell wall loosening and rebuilding. In addition, changes in light signaling perception were found to exhibit cross-talk with hormone-mediated plant growth regulation pathways [38]. $\mathrm{Pt} \mathrm{HY} 5$ was proven to function as a negative regulator that links light and hormone signals. Hence, the decreased accumulation of PtHY5 in a shaded environment could result in an intense auxin signal, which is consistent with published data that shading increases IAA content in $P$. ternata $[22,36]$. Currently, many genes involved in IAA regulation in a shaded environment were identified in a model plant. A previous study reported that the auxin transporter PIN3 and the TF families Aux/IAA and ARF are induced by shade [58]. The expression of PtARF1/19 and PtPIN1/6 were significantly increased in a shaded environment (Fig. 7), which also confirmed that the auxin signal was invigorated by shading. $\beta$-xyloglucan endotransglycosylase (XET) usually mediates cell wall loosening by modifying the xyloglucan chains. In contrast, reversible glycosylated polypeptides (RGPs) function in cell wall synthesis by participating in polysaccharide biosynthesis [59, 60]. Thus, the expression of PtXET and $P t R G P$ were positively correlated with tissue expansion. The upregulation of PtXET and PtRGP genes suggested that cell wall biosynthesis is stimulated by shade (Fig. 7). Besides cell wall reconstitution, cell enlargement also requires many protoplasmic substances. Starch is the main storage-carbohydrate in plants, especially for harvesting organs containing tubers. Sucrose synthase (SUS) is one of the key enzymes that promote the entry of sucrose into various metabolic pathways, and mainly the positive regulation of starch synthesis and plant stress resistance [61, 62]. Hence, upregulation of the PtSUS gene causes starch accumulation in tubers. Overall, we can conclude that the shade environment increases photosynthetic efficiency and reduces ROS accumulation, leading to a decrease in the STF rate. Combinations of polysaccharide metabolic mechanisms including those related to cell wall and starch

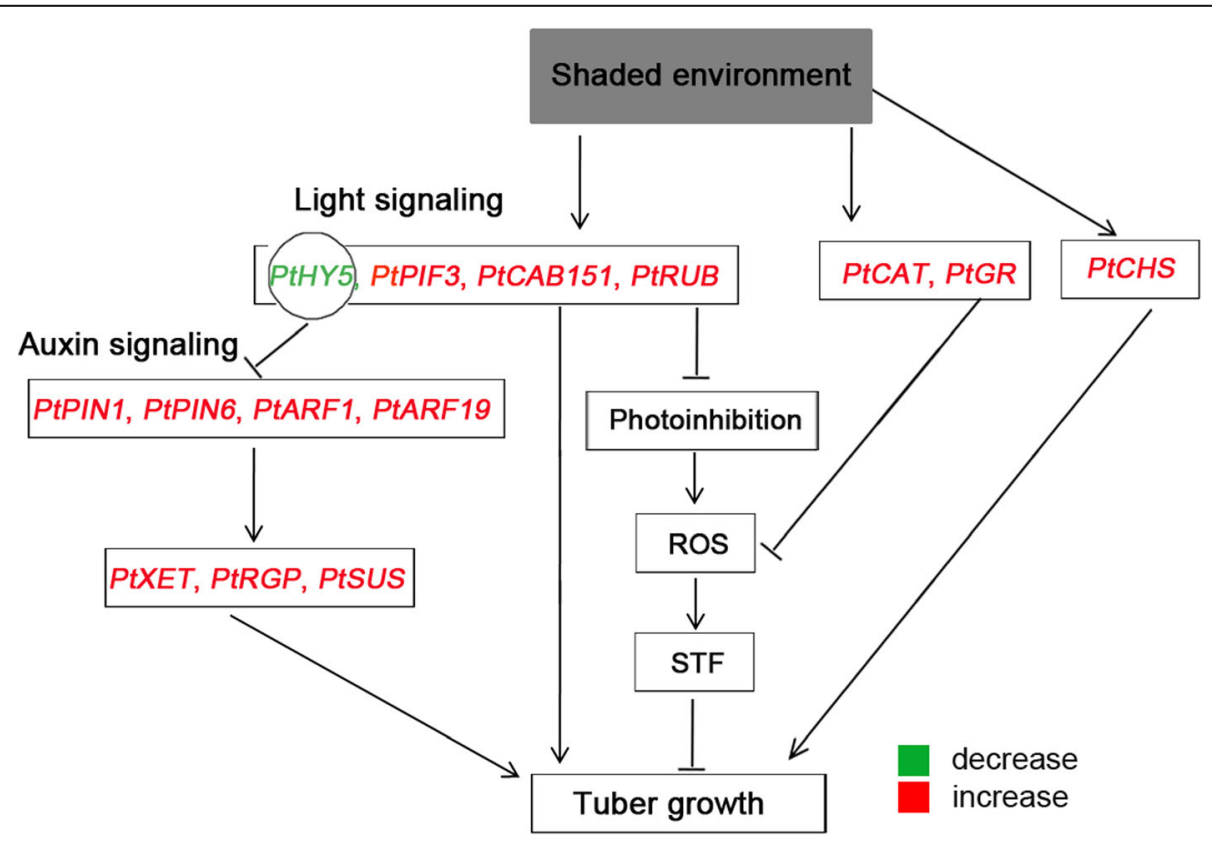

Fig. 8 Putative gene interaction model for tuber growth of $P$. ternata in a shaded environment. A shaded environment suppresses light signaling and increases the photosynthetic efficiency by repressing PtHY5 expression and increasing the transcripts of PtPIF3, PtCAB151, and PtRUB, which not only promote tuber growth directly, but also reduces ROS content via remitting photoinhibition, thereby delaying STF and facilitating tuber growth indirectly. The reduction in PtHY5 expression causes auxin signaling by up-regulating the expression of PtPIN1, PtPIN6, PtARF1, and PtARF19 which further increases the expression of PtXET, PtRGP, and PtSUS, accelerating tuber growth. Additionally, the induction of PtCAT and PtGR in a shaded environment promotes tuber growth by indirectly eliminating ROS accumulation while the up-regulation of PtCHS expedites tuber growth 
were induced by shade. Together, they account for the promotion of tuber growth in the shade (Fig. 8).

\section{Conclusions}

Herein, we generated a comprehensive transcriptome based on the response of $P$. ternata plants to a shaded environment using NGS and MGRT sequencing technologies. A total of 136,163 non-redundant transcripts were generated and 6738 DEGs involved in many KEGG pathways were identified. Sixteen DEGs were selected for expression analysis. Shade was found to improve photosynthetic efficiency and protective enzyme activity, ultimately avoiding photoinhibition-induced ROS damage and causing a delay in STF. Auxin signals and enzymatic genes involved in cell wall reconstruction were upregulated, thereby inducing cell enlargement. These findings add to our knowledge of STF and tuber growth at the molecular level. Moreover, our study provides the first full-length transcriptome resource for $P$. ternata and lays a foundation for further research on this important traditional herb.

\section{Methods}

\section{Plant materials}

The original $P$. ternata, identified by Prof. Jianping Xue (Huaibei Normal University), were obtained from the wild without any specifically permissive requirement and the plant samples were deposited in the specimen room of Huaibei Normal University. It is cultivated in the Experimental Farm of Huaibei Normal University in Huai-

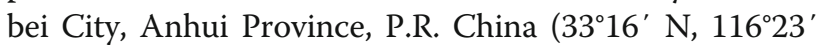
$\mathrm{E}$, altitude, $340 \mathrm{~m})$. No specific permissions were required for samples collection. We stated that the field studies were in accordance with local legislation and no specific licences were required. The tubers of $P$. ternata were planted in the Experimental Farm of Huaibei Normal University in May 2016. When plants grew, approximately 3 weeks after sprouting, half were treated with $90 \%$ shade. The rates of sprout tumble were calculated by analyzing the growth status of $P$. ternata in three random shade- and light-zones after a 30-d shadetreatment. Tuber production per seedling was calculated by measuring the weight of tubers of 30 seedlings in the shade and light zones after $P$. ternata had exhibited STF. Three random shade and light zones were selected for statistical analysis.

\section{Photosynthetic parameters and detection of antioxidant protective enzyme activity}

Leaves of $P$. ternata grown in shade and light zones for $20 \mathrm{~d}$ were used to detect photosynthetic parameters including the content of chlorophyll a and chlorophyll b, $F_{0}, F_{m}, q P$, and NPQ using a previously described method [23]. SOD, CAT, and POD activities in these materials were measured with reference to protocols published previously by our lab [63].

\section{RNA isolation, Illumina CDNA library preparation, and NGS}

P. ternata seedlings were grown in natural light and in a shaded environment and were sampled at 5 and $20 \mathrm{~d}$ (i.e. D5CK, D5S, D20CK, and D20S, respectively). The experiments contained three biological replications. Total RNA of the 12 samples was extracted for transcriptome sequencing using a protocol described previously [64]. Sequencing libraries were generated using a

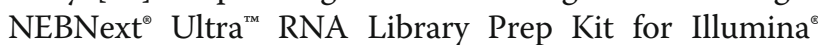
(NEB) following the manual's instruction. NGS was performed at the Novogene Bioinformatics Institute (Novogene, Beijing, China).

\section{PacBio Iso-Seq library preparation, SMRT sequencing and data processing}

Twelve RNA samples were mixed together with equal quantity, and then the mixed RNA was used to prepare SMRT libraries according to the protocol described by Yang et al. [33]. Subsequently, the libraries were sequenced with a Pacific Biosciences RS sequencing instrument. Sequence data were processed using SMRTlink 5.0 software. Circular consensus sequence (CCS) was generated from subread BAM files, and unigenes were obtained using the method reported by Song et al. [64].

\section{Functional annotation and classification}

Gene function was annotated based on the following databases: NR (NCBI non-redundant protein sequences), NT (NCBI non-redundant nucleotide sequences), Pfam (http://pfam.xfam.org/), KOG/COG (http://www.ncbi. nlm.nih.gov/COG/), Swiss-Prot (http://www.expasy.ch/ sprot), KO (KEGG Ortholog database), and GO (Gene Ontology). The Blast2GO program (http://www.blast2 go.com) was used to annotate GO functional classifications. KEGG classification maps were generated based on the retrieved Kyoto Encyclopedia of Genes and Genomes Orthology (KO) information (http://www.genome.jp/kegg). Plant transcription factors in P. ternata were identified using the plant transcription factor database, PlantTFDB 4.0.

\section{Identification and functional analysis of DEGs}

Differential expression analysis was performed with the DESeq $\mathrm{R}$ package (1.10.1) to identify DEGs between samples from the shade and control environments. The expression of DEGs with adjusted $P$-values $<0.05$ based on DESeq were screened as deferentially-expressed, and an absolute $\log _{2}$ (Group1 / Group2) value $\geq 1$ was used as the threshold to identify significant DEGs between 
different groups. GO enrichment analysis of DEGs was performed using the GOseq $\mathrm{R}$ packages. KOBAS software was used to test the statistical enrichment of DEGs in the KEGG pathways.

\section{Validation of DEGs using qRT-PCR}

qRT-PCR assays were performed to validate the 16 candidate DEGs related to STF and tuber growth. cDNA synthesis and transcript abundance analysis were performed with a protocol described previously [65]. Primer sequences are listed in Additional file 8: Table S3. The $P$. ternata $18 \mathrm{~S}$ gene was used as an internal control. Three biological replicates and three technical repeats were used for each gene and sample. The relative mRNA expression level was calculated based on the $2^{-\Delta \Delta C T}$ method.

\section{Statistical analysis}

All assays were conducted in triplicate, the SPSS statistical software 20.0 (SPSS Inc., Chicago, USA) was employed for statistical analyses. The Student's test was applied to test the significant differences between two samples at the level of $P<0.05$.

\section{Supplementary information}

Supplementary information accompanies this paper at https://doi.org/10. 1186/s12870-019-2197-9.

Additional: file 1 Table S1. Overview of sequence data quality obtained from lllumina sequencing.

Additional file 2: Table S2. Summary of final P. ternata nonredundant transcripts generated from SMRT.

Additional file 3: Fig. S1 Functional annotation and categorization of $P$. ternata transcripts. a Venn diagram of NR, NT, GO, KOG and Pfam results for the $P$. ternata transcripts. $b$ Homologous species of $P$. ternata transcripts.

Additional file 4: Fig. S2 Number and family of top 29 TFs predicted by SMRT.

Additional file 5: Fig. S3 Distribution of GO terms for all annotated transcripts in biological process, cellular component and molecular function.

Additional file 6: Fig. S4. KEGG pathways enriched of transcripts. Additional file 7: Fig. S5. Volcano map-analysis of differential expression genes.

Additional file 8: Table S3. Primers used for real-time quantitative PCR.

\section{Abbreviations}

ARF1/19: Auxin response factor 1/19; CAB151: Chlorophyll a/b-binding proteins 151; CAT: Catalase; CHS: Chalcone synthase; DEGs: Differentiallyexpressed genes; flnc: full-Length non-chimeric; FPKM: Fragments per kilobase per million reads; GR: Glutathione reductase; HY5: Elongated hypocotyls 5; NGS: Next generation sequencing; PIF3: Phytochromeinteracting factor 3; PIN1/6: PIN FORMED1/6; POD: Polyamine oxidase; RGP: Reversible glycosylated polypeptides; ROS: Reactive oxygen species; RUB: Rubisco; SMRT: Single-molecule real-time; SOD: Superoxide; STF: Sprout tumble formation; SUS: Sucrose synthase; XET: $\beta$-xyloglucan endotransglycosylase

\section{Authors' contributions}

$T X, Y D$, and JX conceived and designed the experiments. YD and JX collected the materials. TX, HZ, YZ, SW, QC, YZ, JT, AZ, WS, and YD performed the experiments. TX, HZ, QC, YD, and JX compiled and interpreted the data. TX, HZ, YD, and JX wrote the manuscript. All authors reviewed and approved the manuscript.

\section{Funding}

This work was supported by the National Natural Science Foundation of China $(81803665,81573518,31501368)$, the Natural Science Foundation of Anhui Province, China (1408085MC58), the Project of Natural Science Research of Universities in Anhui Province, China (KJ2018A0403, KJ2019B07, KJ2015ZD35, KJ2018B15, KJ2014B21, KJ2016A644, KJ2017A846), and the Innovation Team of Scientific Research Platform of Anhui Province, China (No.KJ2015TD001). The funding bodies were not involved in the design of the study and collection, analysis, and interpretation of data and in writing the manuscript.

\section{Availability of data and materials}

All relevant data are available within the manuscript. The raw bam files of the Sequence Read Archives (SRA) were deposited at the National Center for Biotechnology Information under the accession number SRP215828. The PacBio SMRT reads used for this study were deposited at the National Center for Biotechnology Information Sequence Read Archive under the accession number PRJNA515824. The data can be access with the identifiers search in the web-link (https://www.ncbi.nlm.nih.gov/Traces/study).

Ethics approval and consent to participate

Not applicable.

Consent for publication

Not applicable.

\section{Competing interests}

The authors declare that they have no competing interests.

Received: 5 February 2019 Accepted: 10 December 2019

Published online: 18 December 2019

\section{References}

1. Zhang JY, Guo QS, Zheng DS. Genetic diversity analysis of Pinellia ternata based on SRAP and TRAP markers. Biochem Syst Ecol. 2013;50:258-65.

2. Moon BC, Kim WJ, Ji Y, Lee YM, Kang YM, Choi G. Molecular identification of the traditional herbal medicines, Arisaematis Rhizoma and Pinelliae Tuber, and common adulterants via universal DNA barcode sequences. Genet Mol Res 2016. 15(1):gmr.15017064.

3. Mao ZC, Peng ZS. Progress on research of rapid propagation system of Pinellia ternata. China j Chinese materia medica. 2003;28(3):193-5.

4. Zeng XQ, Peng ZS. Growth and propagation of wild Pinellia ternata in cultivation. China j Chinese materia medica. 2008;33(8):878-83.

5. Iwasa M, Iwasaki T, Ono T, Miyazawa M. Chemical composition and major odor-active compounds of essential oil from PINELLIA TUBER (dried rhizome of Pinellia ternata) as crude drug. J Oleo Sci. 2014;63(2):127-35.

6. Oshio H, Tsukui M, Matsuoka T. Isolation of I-ephedrine from "pinelliae tuber". Chemical pharmaceutical bulletin. 1978;26(7):2096-7.

7. Wei-Feng XU, Zhang BG, Mian LI, Liu GH. Determination of Ephedrine in Rhizoma Pinelliae,Rhizoma Typhonii Flagelliformis and their Processed Products by HPLC. Lishizhen Medicine Materia Medica Res. 2007;18(4):884-5.

8. Ge X, Hao W. Phytochemical properties and quality evaluation methods of Pinelliae ternate. China Pharmaceuticals. 2009;18(9):3-5.

9. Gombodorj S, Yang MH, Shang ZC, Liu RH, Li TX, Yin GP, Kong LY. New phenalenone derivatives from Pinellia ternata tubers derived Aspergillus sp. Fitoterapia. 2017;120:72-8.

10. Xu JY, Dai C, Shan JJ, Xie T, Xie HH, Wang MM, Yang G. Determination of the effect of Pinellia ternata (Thunb.) Breit. On nervous system development by proteomics. J Ethnopharmacol. 2017;213:221-9.

11. Mo XL, Liu YJ, Li T, Peng W, Hu MB, Wu CJ. Extraction optimization and characterization of polysaccharide antioxidants from Pinellia ternata (Thunb) Breit rhizome. Trop J Pharm Res. 2017;16(5):1129-36. 
12. Chen $Y L$, Liu LN, Tang Y, Sun H, Zhou Q. Inhibitive effect of total alkaloids from Pinellia ternate on the proliferation of human hepatic carcinoma cells. J Navy Med. 2014;35(1):5-8.

13. Liu YH, Guo JH, Liu WT, Liang ZS. Research progress on alkaloids from Pinellia ternata. J Northwest University. 2015;43(9):171-7.

14. Lin S, Nie B, Yao G, Yang H, Ye R, Yuan Z. Pinellia ternata (Thunb.) Makino Preparation promotes sleep by increasing REM sleep. Nat Prod Res. 2018:1-4

15. Xu JY, Dai C, Shan JJ, Xie T, Xie HH, Wang MM, Yang G. Determination of the effect of Pinellia ternata (Thunb.) Breit. On nervous system development by proteomics. J Ethnopharmacol. 2018;213:221-9.

16. Lin J, Yao JH, Zhou XW, Sun XF, Tang KX. Expression and purification of a novel mannose-binding lectin from Pinellia ternate. Molecular Biotechnol. 2003;25(3):215-21.

17. Li J, Fang L, Xue J, Sheng W. Effects of Pinellia ternata extracts on inhibiting of oviposition and ovicidal action against Plutella xylostella. China j Chinese materia medica. 2010;35(9):1108-11.

18. Jin S, Zhang $X$, Daniell H. Pinellia ternata agglutinin expression in chloroplasts confers broad spectrum resistance against aphid, whitefly, Lepidopteran insects, bacterial and viral pathogens. Plant Biotechnol J. 2012; 10(3):313-27.

19. Zhang YN, He P, Xue JP, Guo Q, Zhu XY, Fang LP, Li JB. Insecticidal activities and biochemical properties of Pinellia ternata extracts against the beet armyworm Spodoptera exigua. J Asia Pac Entomol. 2017;20(2):469-76.

20. Lu HD, Xue T, Zhang AM, Sheng W, Zhu YF, Chang L, Song YX, Xue JP. Construction of an SSH library of Pinellia ternata under heat stress, and expression analysis of four transcripts. Plant Mol Biol Rep. 2013;31(1):185-94.

21. Xue JP, Wang X, Zhang AM, Chang L. Changes of photosynthesis parameters and chlorophyll fluorescence around sprout tumble of Pinellia ternata under high temperature stress. China journal of Chinese materia medica. 2010;35(17):2233

22. Wang $X$, Xue JP, Zhang AM. Effectsofshading on fresh weight and endogenous of Pinellia ternata tuber. J Nuclear Agricultural Sci. 2008;22(4):514-8.

23. Meng XH, Zhang YJ, Li PI, Yang DF, Zhai YJ. Effect of shading on the photosynthetic pigment and protective enzyme activities in Pinellia ternata leaves. Acta Botan Boreali-Occiden Sin. 2007;27(6):1167-71.

24. Meng XH, Zhang YJ, Zhang HQ, Yan-Changa LU, Yue J. Effects of shading treatment on biological characteristics of Pinellia ternata(Thunb.) Breit. J Northwest A \& F University. 2007;35(3):199-212.

25. Zhao FL, Sun MC, Zhang WJ, Jiang CL, Teng JT, Sheng W, Li MZ, Zhang AM, Duan YB, Xue JP. Comparative transcriptome analysis of roots, stems and leaves of Isodon amethystoides reveals candidate genes involved in Wangzaozins biosynthesis. BMC Plant Biol. 2018;18.

26. Liu Y, Wang Y, Guo F, Zhan L, Mohr T, Cheng P, Huo N, Gu R, Pei D, Sun J. Deep sequencing and transcriptome analyses to identify genes involved in secoiridoid biosynthesis in the Tibetan medicinal plant Swertia mussotii. Sci Rep. 2017;7:43108.

27. Ponniah SK, Thimmapuram J, Bhide K, Kalavacharla V, Manoharan M. Comparative analysis of the root transcriptomes of cultivated sweetpotato (Ipomoea batatas[L.] Lam) and its wild ancestor ( Ipomoea trifida [Kunth] G. Don). BMC Plant Biol. 2017;17:9.

28. Zhang $\mathrm{GH}$, Jiang $\mathrm{NH}$, Song $\mathrm{WL}$, Ma CH, Yang SC, Chen JW. De novo sequencing and Transcriptome analysis of Pinellia ternata identify the candidate genes involved in the biosynthesis of benzoic acid and ephedrine. Front Plant Sci. 2016;7:1209.

29. Du H, Yu Y, Ma Y, Gao Q, Cao Y, Chen Z, Ma B, Qi M, Li Y, Zhao X Sequencing and de novo assembly of a near complete indica rice genome. Nat Commun. 2017:8:15324.

30. Li Z, Xu C, Li K, Shi Y, Xun Q, Zhang J. Phosphate starvation of maize inhibits lateral root formation and alters gene expression in the lateral root primordium zone. BMC Plant Biol. 2012;12(1):89.

31. Kin Fai A, Underwood JG, Lawrence L, Wing Hung W. Improving PacBio long read accuracy by short read alignment. PLoS One. 2012;7(10):e46679.

32. Donald S, Hagen T, Fabian G, Michael S. A single-molecule long-read survey of the human transcriptome. Nat Biotechnol. 2013;31(11):1009.

33. Yang L, Jin Y, Huang W, Sun Q, Liu F, Huang X. Full-length transcriptome sequences of ephemeral plant Arabidopsis pumila provides insight into gene expression dynamics during continuous salt stress. BMC Genomics. 2018;19:717.

34. Ren P, Meng Y, Li B, Ma X, Si E, Lai Y, Wang J, Yao L, Yang K, Shang X. Molecular Mechanisms of Acclimatization to Phosphorus Starvation and Recovery Underlying Full-Length Transcriptome Profiling in Barley (Hordeum vulgareL.). Front Plant Sci. 2018;9:500.
35. Xu QS, Zhu JY, Zhao SQ, Hou Y, Li FD, Tai YL, Wan XC, Wei CL. Transcriptome Profiling Using Single-Molecule Direct RNA Sequencing Approach for In-depth Understanding of Genes in Secondary Metabolism Pathways of Camellia sinensis. Front Plant Sci. 2017:8:1205.

36. Halliday KJ, Martinez-Garcia JF, Josse EM. Integration of light and auxin signaling. Cold Spring Harb Perspect Biol. 2009;1(6):a001586.

37. Franklin KA, Toledo-Ortiz G, Pyott DE, Halliday KJ. Interaction of light and temperature signalling. J Exp Bot. 2014;65(11):2859-71.

38. Xu Z, Peters RJ, Weirather J, Luo H, Liao B, Zhang X, Zhu Y, Ji A, Zhang B, Hu S, et al. Full-length transcriptome sequences and splice variants obtained by a combination of sequencing platforms applied to different root tissues of Salvia miltiorrhiza and tanshinone biosynthesis. Plant jcell molecular biology. 2015;82(6):951-61.

39. Chen J, Tang X, Ren C, Wei B, Wu Y, Wu Q, Pei J. Full-length transcriptome sequences and the identification of putative genes for flavonoid biosynthesis in safflower. BMC Genomics. 2018;19(1):548.

40. Yang $L$, Jin $Y$, Huang W, Sun Q, Liu F, Huang X. Full-length transcriptome sequences of ephemeral plant Arabidopsis pumila provides insight into gene expression dynamics during continuous salt stress. BMC Genomics. 2018;19(1):717.

41. Xue JP, Wang X, Zhang AM, Huang XD, He JQ, Chang L. Effects of shading on photosynthetic characteristics of Pinellia ternata leaves. China Journal of Chinese Materia Medica. 2008;33(24):2896-900.

42. Yan HF, Zhou B, Li YH. Photoreceptors and light signal transduction. Chinese Bulletin of Botany. 2004;21(2):235-46.

43. Hobe S, Fey H, Rogl H, Paulsen H:determination of relative chlorophyll binding affinities in the major light-harvesting chlorophyll a/b complex. Journal of Biological Chemistry2003, 278(8):5912-5919.

44. Andersson I, Backlund a:structure and function of Rubisco. Plant Physiol Biochem2008, 46(3):275-291.

45. Chen M, Chory J. Fankhauser C:light signal transduction in higher plants. Annu Rev Genet. 2004;38:87-117.

46. Carmo-Silva AE, Gore MA, Andrade-Sanchez P, French AN, Hunsaker DJ, Salvucci ME. Decreased CO2 availability and inactivation of Rubisco limit photosynthesis in cotton plants under heat and drought stress in the field. Environmental \& Experimental Botany. 2012;83(17):1-11.

47. Wanzhuo G, Pengfei Q, Junbo D, Xin S, Xiaoling W, Chun S, Weiguo L, Yushan W, Xiaobo Y, Taiwen Y. Transcriptome analysis of shade-induced inhibition on leaf size in relay intercropped soybean. PLoS One. 2014;9(6):e98465.

48. Sonoike K. Photoinhibition of photosystem I. Physiol Plantarum. 2011;142(1):56-64.

49. Liu YF, Qi MF, Li TL. Photosynthesis, photoinhibition, and antioxidant system in tomato leaves stressed by low night temperature and their subsequent recovery. Plant Sci. 2012;196:8-17.

50. Schieber M, Chandel NS. ROS function in redox signaling and oxidative stress. Curr Biol. 2014;24(10):R453-62.

51. Scandalios JG. Oxygen stress and superoxide dismutases. Plant Physiology. 1993;101(1):7-12.

52. Willekens $\mathrm{H}$, Chamnongpol S, Davey M, Schraudner M, Langebartels C, Van MM, Van CW ID. Catalase is a sink for $\mathrm{H}_{2} \mathrm{O}_{2}$ and is indispensable for stress defence in C3 plants. Embo Journal. 2014;16(16):4806-16.

53. Mhamdi A, Hager J, Chaouch S, Queval G, Han Y, Taconnat L, Saindrenan P, Gouia $\mathrm{H}$, Issakidis-Bourguet E, Renou JP, Noctor G. Arabidopsis glutathione reductase1 plays a crucial role in leaf responses to intracellular hydrogen peroxide and in ensuring appropriate gene expression through both salicylic acid and jasmonic acid signaling pathways. Plant Physiology. 2010;153(3):1144-60.

54. Wimalasekera R, Tebartz F, GFE S. polyamines, polyamine oxidases and nitric oxide in development, abiotic and biotic stresses. Plant Science An International Journal of Experimental Plant Biology. 2011;181(5):593-603.

55. Wei S, Xue JP, Zhang AM, Zhang HT. Structure of cell and histology around sprout tumble of Pinellia ternata. Guihaia. 2009;29(3):390-2.

56. Zhou B, Wang Y, Zhan YG, Li YH, Kawabata S. Chalcone synthase family genes have redundant roles in anthocyanin biosynthesis and in response to blue/Uva light in turnip (brassica Rapa; Brassicaceae). Am J Bot. 2013;100(12):2458-67.

57. Kim SH, Mizuno K, Sawada S, Fujimura T. Regulation of tuber formation and ADP-glucose pyrophosphorylase (AGPase) in sweet potato ( Ipomoea batatas (L.) lam.) by nitrate. Plant Growth Regul. 2002;37(3):207-13.

58. Ding Z, Zhang Y, Xiao Y, Liu F, Wang M, Zhu X, Liu P, Sun Q, Wang W, Peng M. Transcriptome response of cassava leaves under natural shade. Sci RepUk. 2016;6:31673.

59. Campbell P, Braam J. Xyloglucan endotransglycosylases: diversity of genes, enzymes and potential wall-modifying functions. Trends Plant Sci. 1999:4(9):361-6. 
60. Langeveld SM, Vennik M, Kottenhagen M, Van Wijk R, Buijk A, Kijne JW, de Pater S. Glucosylation activity and complex formation of two classes of reversibly glycosylated polypeptides. Plant Physiol. 2002; 129(1):278-89.

61. Ruan YL: Sucrose Metabolism: Gateway to Diverse Carbon Use and Sugar Signaling. Annual Review Of Plant Biology, Vol 65 2014, 65:33-67.

62. Tauzin AS, Giardina T. Sucrose and invertases, a part of the plant defense response to the biotic stresses. Front Plant Sci. 2014;5:293.

63. Xue JP, Ding Y, Zhang AM, Hu CQ. The change of activity of protective enzyme around sprout tumble of Pinellia ternate under high temperature stress. China j Chinese materia medica. 2004;29(7):641-3.

64. Song H, Yang MJ, Yu ZL, Zhang T. Characterization of the whole transcriptome of whelk Rapana venosa by single-molecule mRNA sequencing. MAR GENOM. 2019;44:74-7.

65. Xue T, Yue EK, Chao EK, Su Y, Zhang WJ, Zhu YF, Teng JT, Xue JP. Identification and expression of Ga-related genes associated with in vitro micro-tubers formation in Pinellia Ternata. Acta Sci Pol-Hortoru. 2018;17(6): 85-94.

\section{Publisher's Note}

Springer Nature remains neutral with regard to jurisdictional claims in published maps and institutional affiliations.

- fast, convenient online submission

- thorough peer review by experienced researchers in your field

- rapid publication on acceptance

- support for research data, including large and complex data types

- gold Open Access which fosters wider collaboration and increased citations

- maximum visibility for your research: over $100 \mathrm{M}$ website views per year

At $\mathrm{BMC}$, research is always in progress. 